\title{
Visualizing demographic evolution using geographically inconsistent census data
}

\author{
Fabio Dias and Daniel Silver
}

\begin{abstract}
We propose a visual analytics system that enables the exploration evolutionary patterns in geographically inconsistent data, removing the need for harmonization of the geographical regions into a common geometry, a time consuming and error-prone process that is currently used in virtually all longitudinal analysis of geographical data. This work also includes incremental developments in the representation, clustering, and visual exploration of region-based geographical data. While we leverage the well known context of census data, our proposal is suitable for any region-based data. The method enables an easier identification and understanding of the demographic groups present in a city and their evolution over time. We present the feedback of experts in urban sciences and sociology, along with illustrative scenarios in the USA and Canada on the decennial censuses between 1970 and 2010.
\end{abstract}

\section{INTRODUCTION}

$\mathrm{U}$ RBAN sciences are blooming thanks to a renewed interest in understanding and improving the urban environment. Visual analytics is following this trend, fueled by new public datasets that encompass progressively more of our daily lives [1]. There is no shortage of methods to explore mobility patterns [2], social media [1], traffic [3], and so on, providing experts, planners, policy makers, and the general population with deeper insights about their cities.

These new datasets usually contain GPS coordinates for the records, leading to point-based data. Combined with the corresponding timestamps, this data is easily suitable for longitudinal analysis. But most demographic datasets are region-based, where the measurements are associated with pre-defined regions, not only for an additional level of privacy protection, but because some measurements only make sense over a defined area. Census data is a classic example of this format, with datasets available from 1790 onward for the US [4]. Despite this unmatched temporal availability, longitudinal analyses of census data are often restricted in time, especially when smaller tabulation areas are considered, such as census tracts (CT) or dissemination areas, which evolve to reflect changes in population density, leading to geographic inconsistencies across time, and the traditional time-series based approach is no longer viable.

However, these analyses are necessary to understand the urban environment. Indeed, two different regions can have similar average income for a given year, while one is experiencing a process of economic improvement and the other one impoverishment. A single snapshot cannot be used to identify gentrification, migration, education changes, or any of the relevant processes that happen over time.

To overcome these inconsistencies, the traditional approach is the geographical harmonization of the data, the interpolation of the measurements into a common set of

- F. Dias is with the Department of Mechanical \& Industrial Engineering, University of Toronto, Toronto, ON, M5S $3 G 8$.

E-mail:fabio.dias@utoronto.ca

- D. Silver is with the Department of Sociology, University of Toronto, Toronto, ON, M5S 2J4.

E-mail:dsilver@utsc.utoronto.ca

Manuscript received MMMM DD, YYYY; revised MMMM DD, YYYY. regions [5], [6], |7], so that each variable can be represented using time-series. This is laborious work that inevitably introduces some amount of error [8], even when additional data is provided [9]. Nevertheless, this step is considered mandatory in the current literature: "(...) tract-by-tract comparison is not possible unless data from 2000 is interpolated to 2010 boundaries (...)" |10].

The main contribution of this application paper is an visualization-based alternative to the geographical harmonization, a combination of established graph based processing and information visualization techniques allowing tractby-tract comparison, the identification and visualization of patterns of demographic evolution without geographic harmonization, effectively removing one of the most challenging problems in longitudinal demographic analysis. We also include illustrative scenarios and our prototype is available at http://uoft.me/piccard, including more than forty regions in the US and Canada. The source code is publicly available at https://github.com/fabioasdias/piccard

\section{Related Work}

Since our problem encompasses several fields, we divided this section into specific sub problems: longitudinal demographic studies, describing the traditional approach to perform longitudinal studies; data representation, exploring how evolving geographic data can be represented for processing; Data clustering, briefly reviewing existing clustering methods; and cluster characterization, exploring how the clusters can be visually summarized.

\subsection{Longitudinal demographic studies}

Census data is used not only to discover demographic patterns [11], but to correlate demographic characteristics to other measurements [12]. However, longitudinal studies are rare: "(...) One of the most challenging and fascinating areas in spatial statistics is the synthesis of spatial data collected at different spatial scales(...)" [13].

While CT level data is readily available for the US since 1910 [4], most studies consider the period between 1970 
and 2010, using pre-harmonized data [4], [5]. Despite the inherent errors [6], [8], this dataset became the standard source for longitudinal demographic data, with similar efforts appearing in other countries [7], [14], [15]. This result was significant for the field, but it also restricts the usable data, since new datasets need to be similarly processed.

Another option considers the use of grid data [10], [16], where small rectangular areas are used, in an approach similar to satellite imagery. Beyond the increased spatial accuracy, this approach does not require complex harmonization when new data is considered. However, demographic data is usually not available in this format, especially from older sources, and the conversion from tabulation areas can introduce significant errors.

In the proposed methodology, we avoid the harmonization by considering each measurement using its actual geographic region. It does not require the regions to be consistent across time because they are already represented as different entities.

\subsection{Data representation}

Most data is represented in tabular form, where the rows and columns have coherent definitions. For example, consider a table with rain measurements over time, with the rows representing different locations and the columns different times. This representation can also be interpreted as a collection of time-series, one for each location. Geographic data followed this format, only including an additional field that describes the associated geographic area. Following the example, the data would now represent the amount of rain for a given region and time. As long each region remains the same, the data is coherent and can be interpreted again as a collection of time-series.

In the proposed method, we remove the requirement for consistency in the measurement regions by leveraging a graph-based representation, where each region in time corresponds to a different node. Instead of a collection of timeseries, the data is represented as a dynamic graph. Graph based representation of geographic information is fairly well explored in the literature, as a basis for topological methods for event detection [17], leveraging signal processing on graphs [18], [19] to find patterns and outliers [20], [21], [22. Graphs are well suited to represent trajectories as well [2], [3], [23], allowing the use of graph visualization methods [24], [25].

Graphs were used to represent census data for clustering purposes before [21], [26], but these works did not explore temporal evolution, where graphs are particular powerful as they allow a natural representation of inconsistent regions, with both spatial and temporal connections. Note that there are other possible representations that have similar properties, but we adopted graphs to allow the use of the existing literature and methods.

\subsection{Data clustering}

Data clustering is one of the elementary processes for data analysis, simplifying the data into a smaller number of homogeneous sets that can be interpreted in the same way.
While there is no shortage of contributions for this problem [27], most applications still rely on k-means [28], [29] and, to a lesser extent, Self Organizing Maps [30], [31].

However, a method for geographic data analysis should not ignore the geographic component of the data. One straightforward option, for agglomerative methods [32], is to consider only nearby clusters for merging [33], which can also be done for k-means [34]. Alternatively, the spatial distance could be directly added to the inter-cluster metric [33] via a mixing parameter, which adds flexibility to the method, but introduces the problem of finding the correct application-dependent values.

Indeed, one crucial step in most clustering algorithms is the definition of the number of clusters. We sidestep this problem by considering hierarchical methods [35], where the result is not a partition of the data, but a tree of partitions. This approach is interesting for interactive methods, because it allows the user to change the number of displayed clusters with minimal processing. Since our data is represented as a graph, one option would be the watershed cuts algorithm [36], inspired by the well known image processing segmentation and equally prone to over segmentation. Considering that the processing time is also a relevant factor, we opted for an heuristic variation of the maximum weighted matching algorithm called sorted maximal matching [37], which merges clusters based on the weights of the edges between pairs of clusters.

\subsection{Cluster characterization}

Visually representing evolving spatial data is a challenging old problem [38], |39|, |40|, |41|. Most geographic data is naturally bi dimensional and maps work well in this case [41], [42], but the temporal dimension cannot be so naturally represented. One straightforward option is to leverage tridimensional plots [43], [44], but this can lead to visual obstructions or scaling problems unless a tridimensional display device is used. Animation can also be explored in some specific cases [45], but it is not a general approach. Glyphs can also be used [46], [47], but this may lead to cluttering when many small regions are present. A simpler, well adopted, option is to display a map that corresponds to a subset of the temporal information, allowing the user to change the time with an associated control [1], [17], [20], [22]. Small multiples can be used [2], but only when there are few temporal snapshots. However, none of these options is suitable to represent many variables at the same time.

Using data clustering, we can represent the region's cluster instead of all the its variables [2], [20], [22]. While this simplifies the geographic portion of the visualization, it introduces the problem of how to summarize the contents of each cluster. One traditional approach is to use parallel coordinates plot [48], [49], [50], [51], but these they can get cluttered representing similar clusters over several variables. Further, for demographic applications, the clusters are usually strongly characterized by a small subset of values [29], [30|. Therefore, in the proposed method, we identify the variables that are most relevant to the characterization of each cluster. The distribution of values on that variable is then represented using a boxplot, a well known statistical plot displaying basic properties of the distributions. 


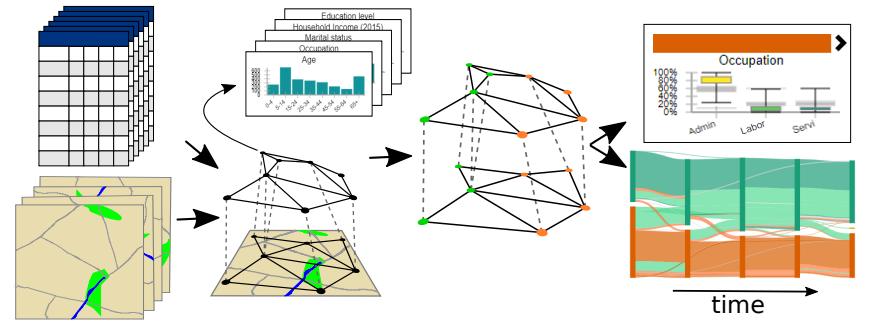

Fig. 1. Overview of the proposed method. A graph is generated combining the original census data, encoding the changing geographical information. The graph is partitioned into an hierarchy [37. The characteristics and evolution of the clusters are then visually represented.

\section{VISUALIZING THE DEMOGRAPHIC SPATIO- TEMPORAL EVOLUTION}

Beyond the objective of allowing the study of inconsistent data, our method includes incremental developments in most steps of the analysis, from data representation to the visualization method for the clusters. Figure 1 presents an overview of the processing steps of the proposed method.

\subsection{Census methodology and data representation}

Census data is disseminated in a tabulated form for aggregation areas: whole country, state/province, metropolitan region, and so on. To provide as much detail as possible, we focus on the smallest region with available data: census tracts (CT). They are usually defined to maintain the anonymity of the population, leading to a population count in the order of thousands in densely populated areas. Physical barriers are usually adopted as borders, so these regions can change because of new roads, construction or removal of high density buildings, and so on. Some census entities also consider demographic characteristics, aiming to establish the CTs as a cohesive unit. Therefore, CTs are the least geographically stable tabulation area.

Each CT is associated with a series of variables, with counts derived from the census questionnaires, covering several aspects of the demographic characteristics of the population. Some questions allow for multiple choices or open answers, that are then tabulated into the most frequent categories. Since the census is often used to direct government initiatives, which variables are measured/disseminated is dependent on administrative interests, the general understanding of the population, and current customs. For instance, income is disseminated with a finer tabulation in the lower portion than on the higher.

To match these variables over time and allow for direct comparison across different census years, we aggregated similar ones (e.g. White, Black, Asian, Other) into aspects (e.g. Race), encoding the distribution of that facet of the population. In this convention, we refer to the composing parts of an aspect as a part or the traditional variable. Internally, the aspects are represented using normalized histograms. This normalized representation is crucial for the comparison between inconsistent regions.

In our graph based representation, each CT of each census year is represented as a node, and edges are placed between nodes if the corresponding CTs share geographic borders in the same year. Further, edges are placed between nodes if the corresponding CTs belong to sequential years and there is geographical overlap between them. This approach leads to a single graph representing the whole spatio-temporal space of the data. Our objective then becomes to identify partitions of this graph such that the nodes of each partition are more similar between themselves than to the other nodes. This representation is not the only option, nor unique, but it allows the use of existing graphbased methods for the other steps.

\subsection{Geographic content clustering}

To partition the graph we must first establish a distance function between the nodes, measuring the data similarity. This similarity is then associated with the edges, leading to a weighted dynamic graph. Every node has a collection of histograms, each representing the distribution of certain aspect in the population.

Let $G=(V, E)$ be a graph, where $V=\left\{v_{1}, v_{2}, \ldots, v_{n}\right\}$ is the set of nodes and $E=\left\{\left(v_{i}, v_{j}\right), i \neq j\right.$ and $\left.i, j \in[1, n]\right\}$ is the set of edges. A function $H$ associates each node to a set of $K$ histograms. We define the distance $D$ between two nodes $v_{i}$ and $v_{j}$ as:

$$
D\left(v_{i}, v_{j}\right)=\sum_{k \in[1, K]} w_{k} d\left(H_{k}\left(v_{i}\right), H_{k}\left(v_{j}\right)\right)
$$

where $d$ is a distance metric between histograms and $w$ is a sequence of non-negative weights associated with each aspect, $\sum_{k \in[1, K]} w_{k}=1$. While any histogram metric can be used, we adopted a euclidean distance between the vectors, because it led to reasonable results with reduced computational cost. Therefore the distance between two nodes is defined as the weighted average distance between its associated histograms, where the weights can be adjusted by the user.

Once the distances are associated to the edges, we use watershed cuts [36] to create an initial clustering, which is then refined into a hierarchy using the Sorted Maximal Matching (SMM) [37] with median linkage. The initial watershed step is performed to create an initial clustering and reduce the running time of the SMM. For completeness, we briefly review this method, but we refer the reader to the original paper [37] for more details, including a complete performance evaluation using several metrics.

We included two application-specific parameters: the maximum number of clusters to be shown and a distance threshold. Contrarily to the original SMM, which merges all clusters in all steps, we only merge two clusters where the distance is above the threshold after we reach the maximum number of displayed clusters. Without this restriction, significantly different clusters would be merged early, leading to increased intra cluster variance and the disappearance of small outlier regions. Further, after the maximum number of clusters is reached, we create one step of the hierarchy for each merge, leading to a binary partition tree. In this structure we can directly access a result with an arbitrary number of clusters.

Each resulting cluster is contiguous in the graph. This means that two similar, but non-contiguous, sets of CTs will be classified into two different clusters, which can 
be counter-intuitive. To overcome this issue, we augment the graph with two new edges per node from a nearest neighbors graph [52] using only the distances between the histograms. These edges connect nodes with similar content, if they are not already connected, providing a path for the algorithm to group similar nodes. Theoretically, adding more of these content based edges could be used to decrease the impact of the spatio-temporal edges, controlling the balance between content and topology in the result. In practice, the effect is dependent on the data itself, and the results are not consistent, or predictable, across different cities. We fixed it at two edges because it was the lowest number that empirically led to consistent clusters, but we believe that this idea warrants further investigation, as an alternative to mixing parameters in the distance metric [33].

\subsection{Cluster characterization and variable relevance}

The composition of each cluster is determined by simple statistical measures, considering each aspect separately. We compute the minimum, maximum, median, $25 \%$, and $75 \%$ quantiles for each part of each aspect for all clusters in the hierarchy. While interpreting these values is more complex than interpreting just the average, they provide far more information about the underlying distribution.

We also use these statistical measurements to discover what characterizes each cluster, that is, what makes it different from the others. We define the relevance of a part of an aspect based on the distance between the interquantile ranges (IQR) of the clusters in the same hierarchical level. If the IQRs overlap for all clusters, that variable is not relevant to the characterization of the cluster, but if the IQRs are distant, it means that this specific range of values is something that only occurs in this cluster.

\subsection{Clusters and trajectories}

While the partition of the data into different clusters helps the user to understand what groups exist and where they are, we are also interested in the evolution of these groups. We introduce the concept of trajectories, composed by regions classified into the same sequence of clusters over the considered period. This enables direct access to regions that evolved in the same manner. While the interface provides access to data by individual census tract, the trajectories are the main unit of exploration in this work.

\subsection{Colors}

As illustrated by Figure 3 and further explored in the next subsection, our proposed interface heavily relies on color to express cluster-related information. We adopted this convention because colors can be used in all our visual tools in a coherent manner. However, this also introduced significant challenges. The first is the limit on the number of clusters that can be visually represented. We limited the number of clusters to eight because this was the largest number of colors that we could reliably use, derived from the 8-class Dark2 set from ColorBrewer [53].

While we can reasonably limit the number of clusters, there are far more possible trajectories. And the color associated with each trajectory should bear some resemblance
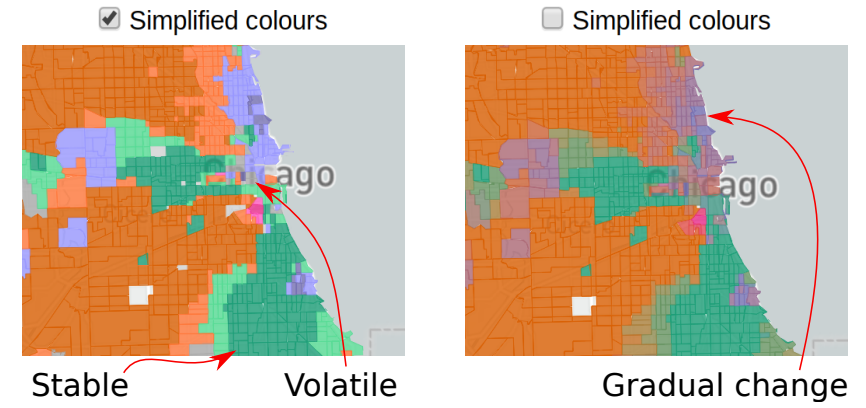

Fig. 2. Different color schemes for Chicago with four clusters. Left: simplified, right: average color.

to the clusters included in it. Therefore, we were left with a conundrum: Should we associate each trajectory with a unique color, which the user probably cannot distinguish, or should we use a reduced set of colors and associate the same color to different trajectories? Since there are advantages and disadvantages for each of those options, we adopted both. The user can control which color policy is used via a checkbox in the configuration panel, on the top left of the interface.

By default, the interface adopts a simplified color scheme, where a trajectory is painted in the same color of a cluster if the regions were associated to that cluster for all times; in a slightly less saturated version of that color if the regions were associated to that cluster for the simple majority of the time, and gray otherwise. In this mode, the colors will mostly represent stability, immediately identifying the regions that were consistently associated with each cluster. It also easily identifies volatile regions, painted gray.

When this simplified color scheme is disabled, each trajectory will be painted using the average of the colors of the involved clusters, in the LAB color space. In this mode, the map becomes more similar to a heatmap, where stronger presence of a color indicates more temporal affinity to the cluster. Volatile regions will also tend to be displayed in gray, as the average of three or more colors.

While both approaches will use more than eight colors, in practice this is not as significant because most cities can be explained using less than eight clusters. In fact, articles in the literature usually employ from two to five, which are fairly stable across time. For the more dynamic scenarios, user interaction can be used to alleviate the shortcomings of both approaches.

\subsection{User interface}

The initial interface is illustrated in Figure 3 Since demographic data can be nuanced, with intricate interconnections, we decided against validating the interface using a synthetic dataset, considering instead data from the Chicago region between 1970 and 2010, using previous published studies as corroboration. This region is known for its entrenched racial divide and the emergence of a 'young urban' population with a higher education level [29], [30]. More details about this dataset are presented in Section 4 .

The configuration panel, on top left in Figure 3, displays which aspects were used and their weights (following Equation 11. It also includes other configuration options that can be altered without re-processing the data, such as the 


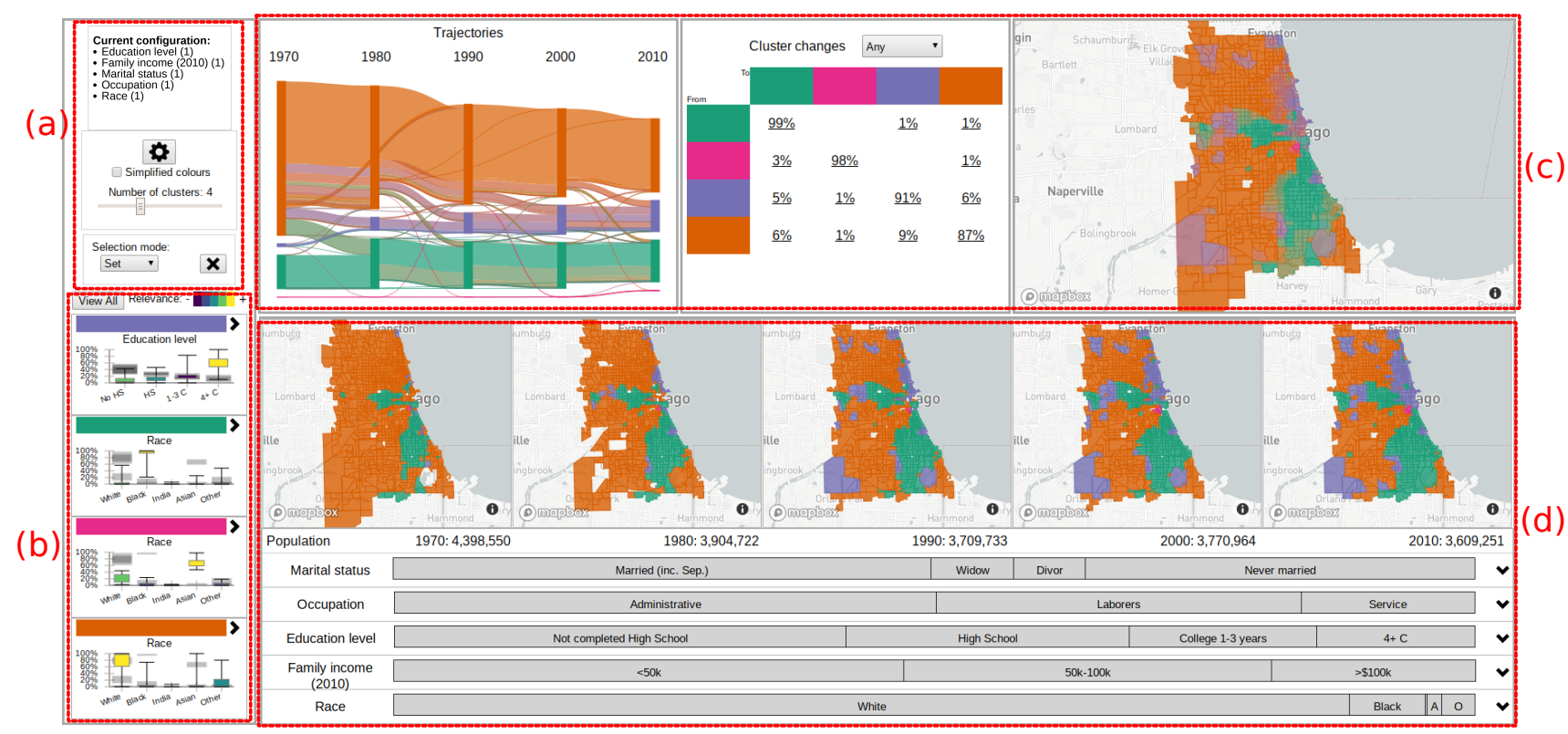

Fig. 3. Initial interface of our method showing the demographic evolution of Chicago. (a): Configuration panel with the current clustering parameters and controls. (b): Cluster overview illustrating the most relevant aspect for each cluster. (c): Trajectories overview and the general evolution of the population, geographical information, and how it changed. (d): Details of the selected trajectories, including precise geographic locations, population numbers, and the composition of the aspects.

number of clusters and the color option. The gear button allows access to the other configuration options that do require further processing, such as changing location, aspects, and weights. This panel also includes the configuration of the selection mode for the trajectories, which allows the user to set, add, or remove the next selected trajectories to the current selection. This feature enables the analysis of complex sets of trajectories.

The cluster overview panel, on the bottom left in Figure 3. displays a brief summary of each cluster, based on the distance between the IQRs, as detailed in Section 3.3 The View all button opens a new panel where all aspects are represented, while the chevron at the side of the color lets the user expand each cluster separately. While the standard approach to represent cluster characteristics is to use parallel coordinates [50], [51], this representation occupies screen space proportional to the number of variables and can get cluttered with a higher number of clusters, or when the clusters are not well defined for multiple variables. To save space and leverage the familiarity scientists have with statistical tools, we opted to use boxplots to properly convey the distribution of each variable in the current cluster. However, a simple boxplot would not include information about the other clusters, forcing the user to mentally compare them to find what is relevant.

We adopt an enhanced version of the traditional boxplot, which includes the minimum, maximum, 25\% and $75 \%$ quantiles for the current cluster, but also the IQRs for the other clusters, in slightly larger and faded black rectangles. We also color the current IQR according to its relevance. While there might be some degree of similarity between the color schema for relevance and for trajectory identification, none of the experts consulted reported confusion. Indeed, one expert reported confusion regarding the grey rectangles that represent the IQRs for other distributions when they

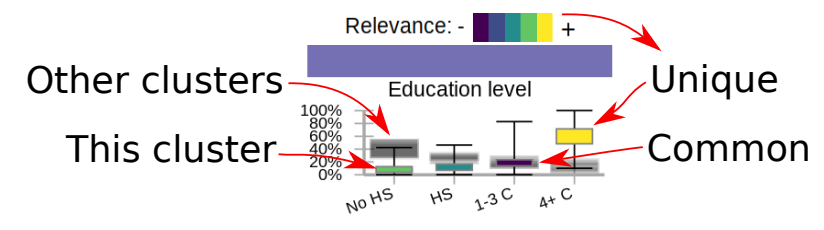

Fig. 4. Enhanced boxplot of the clusters' characteristics allows a quick comparison to the other clusters.

are not colored; when that variable is not relevant to the characterization of the cluster. These simple changes allow the user to easily understand the composition of the cluster and how it relates to the others. Violin plots [54] could also be used, providing more information about the shape of the distribution, but with increased potential for obstructing the representation of the other clusters.

For instance, the boxplot that summarizes the purple cluster illustrated in Figure 3 , detailed on Figure 4, illustrates that this cluster is best defined by the proportion of the population with four or more years of college. The user can quickly see that this is relevant because the corresponding IQR is colored with the highest relevance present in the legend. It is also clear that, while this cluster includes CTs that have between $10 \%$ to $90 \%$ of people in this variable, approximately, half of them have about $60 \%$ of the population with four or more years of college. Since all the other IQRs are well separated, this is a defining characteristic of this cluster. Conversely, the proportion of the population with one to three years of college is not relevant, as indicated by black fill in the rectangle representing the IQR of this cluster, in overlapping position with the rectangles of the other clusters. By clicking on the colored bar above the boxplot, the user can select all trajectories that contain this cluster at any point in time. 
The other clusters identified on Figure 3 correspond to higher concentration of people that identify as Black in the green cluster, people that identify as "Asian, Hawaiian, other pacific islander" in the pink cluster, and people that identify as White in the orange cluster. From these plots, it is clear that the city is indeed racially divided [29], with several CTs that are almost exclusively occupied by people of the same racial category.

The trajectories overview aims to convey basic information about the trajectories, where they are, and what changes are involved. This is done using three sub panels. The first, on the left, contains a Sankey diagram illustrating the evolution of the clusters over time. The widths are proportional to the population involved, the colors follow a policy detailed in Section 3.5. A stacked graph could also be used to represent the proportions of each cluster [20| with less clutter, since the transitions between clusters would not be represented. However, this is only viable if more temporal steps are available, making the plot smoother. Another option to remove clutter is to remove portions of this plot when trajectories are selected, but this would change the layout and compromise the user's mental map.

In our example in Figure 3, the total population of Chicago is decreasing. Additionally, the orange and green clusters contain most of the population and are fairly stable over time. The pink cluster is small and mostly stable. The purple cluster is increasing, mostly by incorporating areas that were previously orange. Since the purple group corresponds to the emergent 'young urban' group, this corroborates the findings of Delmelle [29], |30|. This diagram can also be used to select specific trajectories, by clicking on the bands, or all trajectories that contain a specific cluster at a specific time, by clicking on the rectangles.

In the next panel, illustrated in the top middle of Figure 3, is a transition matrix between the clusters. It indicates the percentage of the population whose area changed between each pair of clusters. This kind of table can be found in the related literature [29], so it is familiar to the advanced users. It not only informs the proportional changes, but allows the selection of the corresponding trajectories for further analysis.

Contrary to the trajectories plot, this representation is more Markovian, where only the current and next state are considered. This panel also enables easier access to trajectories with specific changes, by clicking on the corresponding percentage values. The combo box allows the user to refine the transitions, from 'Any', which includes all transitions between years, to specific transitions, to changes from the first year to the last year. In this example, approximately $99 \%$ of the population in areas classified as green were also in areas classified as green in the next year, while $1 \%$ changed to purple at some point and another $1 \%$ to orange. The total is over $100 \%$ due to rounding errors. Regions changed from the orange to the green cluster for $6 \%$ of its population, $1 \%$ to pink, and $9 \%$ to purple. This further corroborates the fact that most of the growth of the purple cluster came from the orange cluster. Additionally, the lack of transitions is also relevant, for instance, no CT changed from majority of Black population (green) to Asian population (pink), and no CT with significant Asian population had significant increase in education levels (purple).
The panel in the top right of Figure 3 is a map of the region under analysis, summarizing the geographical evolution of the clusters over time. The colors are derived from the clusters involved in each trajectory as detailed in Section 3.5, which are consistent across the linked views.

The bottom part of the interface contains the details for the selected trajectories, or for the whole city if nothing is selected, as illustrated in Figure 3 . This panel contains two main regions: the small multiple maps, depicting the clusters at each year, and the stacked bar plots that summarize the overall composition of these regions. Some finer localization information is lost using small multiples, such as small border changes, but that information is available at the larger map. All the maps are linked with synchronized navigation, and the use of small multiples allows the exploration of each temporal census individually, and its comparison to the others, with minimal interaction.

In this example, the maps show the transition from orange to green and purple in several regions over time. Clicking on a region in these maps will bring up a new panel with the original census data of this specific region. The actual population numbers are below the maps, and they confirm the notion provided by the Sankey diagram that the total population is indeed decreasing.

Each aspect is represented by a stacked bar plot, where the width of each rectangle corresponds to the average percentage of that variable over the considered period. We chose stacked bar plots to represent the composition of the regions because they can accurately and succinctly inform the proportions of each aspect, without any interaction. In this case, about half of the people in Chicago in the considered period are married, and the percentage that are Widowers or Divorced is roughly similar. About half of the population work in Administrative jobs, a third never completed high-school, approximately half have gross family income below 50,000USD per year. The vast majority identify as white. Placing the mouse over one of the bars will open a small panel with the temporal evolution of that specific variable, and clicking on the chevron on the right side expands the corresponding aspect, showing details of the temporal evolution of each variable and also the corresponding IQRs for the whole city.

\section{Illustrative scenarios}

We used decennial census data from the United States [4] and Canadi tabulated by CTs, from 1970 to 2010. The prototype allows access to 40 regions, 28 in the US and 12 in Canada. Due to the high number of CTs, New York City was split into its boroughs.

We used five aspects for the USA: Education level, Family income, Marital status, Occupation, and Race; and seven for Canada: Age, Education level, Home language, Household Income, Marital status, Occupation, Place of birth, and Religion. While our method does not require geographic harmonization, it requires matching variables over time. The supplementary material contains the details of which census columns were used for each aspect. Income is slightly inaccurate, even though we did correct for official

1. http://datacentre.chass.utoronto.ca/census/ 
inflation. We grouped the original ranges into three larger ranges, but they do not match precisely.

\subsection{Chicago}

We selected a region loosely following the administrative borders. The demographic composition is well explored in the literature, with reports of racial divide and gentrification [29], [30], [55]. While the definition of gentrification is still unclear and out of the scope of this paper, we associate gentrification with higher education and income levels.

The initial state of the prototype is illustrated in Figure 3 . and its findings are explained in Section 3.6. where the racial divide is clear. Starting from this initial state, the specific workflow used to identify the existence and details of the gentrification process are illustrated in Figure 5 For the users, the first step is to identify the compositions of each cluster from the boxplots, so orange is associated with majority of White population, green with majority Black, and purple with higher proportion of four years of college or more (high education level). The expanded version of the boxplots for the purple cluster shows a higher income level and majority of occupations in administrative jobs, therefore the purple cluster identifies gentrified regions.

The trajectories plot illustrates the process of gentrification, progressively absorbing regions from the orange cluster (White). This corroborates results from the literature reporting that Black neighborhoods are less likely to gentrify [55]. Moreover, this process is unlikely to be reversed, as indicated by the limited number of trajectories leaving the purple stream. Next, we select the region that is gentrified in 2010, by clicking on the corresponding rectangle in the trajectories plot, updating the information on the maps and the details portion of the interface.

The corresponding CTs are highlighted in the maps, where the spatial pattern is clear, corresponding exactly to previous findings in the literature [55]. Further, we can also identify the regions that gentrified earlier, with an stronger purple hue, compared to newer regions, where the orange and green colors are still present. This also indicates from which clusters they belonged before gentrifying. In the details portion of the interface, the order of the aspects was updated to reflect the order of relevance considering only the selected region. The most relevant aspect is the Education, specifically "Four or more years of college", illustrated in the rightmost portion of Figure 5. which is increasing for the whole city (grey band), but faster and to a higher level in this region (black band). Indeed, while the IQR for the city goes from $11 \%$ to $45 \%$, the IQR for this region goes from $55 \%$ to $77 \%$. However, there are portions of this region with significantly lower or higher proportions, as indicated by the dotted black lines representing the minimum and maximum for the selected region.

\subsection{Toronto}

We considered a region that is approximately the administrative border of the current city of Toronto and all seven available aspects with equal weights. The results are summarized in Figure 6 considering eight clusters.

The population with low percentage of University degrees is represented in orange, mostly anglophone population in green, Asian immigrants in yellow, high percentage of income in the highest bracket in purple, high percentage of Jewish people in light green and brown, high percentage of Eastern Non-Christian religion in pink, and high concentration of single people in dark gray. From the trajectories plot, we can see that Toronto is more dynamic than Chicago, with one cluster constantly shrinking. In the 1970s, the city was divided into four clusters: low number of university degrees, Jewish population, majority anglophones, and high income. Interestingly, the more recent clusters that absorbed regions from the orange cluster have similar education profiles and are differentiated by other aspects. In this sense, the city is growing diverse, changing from a common low education profile to a higher level of education with more diversity in religion (pink) and immigration (yellow).

Indeed, the influx of Asian population is visible starting in the 1980s and building thereafter, leading to the yellow and pink clusters. While both include a high percentage of people born in Asia, the pink is more defined by religion, with low percentage of university degrees, and contains the lowest percentage of people in the highest income bracket for these clusters; the yellow is less defined by religion, and has higher education and income, geographically corresponding to the Markham region, know for its Chinese population. A similar division also happens for the two Jewish clusters, where the light green cluster has lower education and income levels than the brown cluster. The purple cluster of high income is somewhat stable. This cluster includes the Bridle Path neighborhood, known for its wealthy population, until 2011. In 2011 it was classified into the yellow cluster of Asian immigration, since about $35 \%$ of the population for this CT were then born in Asia. The income distribution did not change, with $85 \%$ of the population with an income of $90 \mathrm{k}$ CAD or more.

The most significant indicator of Toronto's dynamism is the presence of grey regions on the simplified color map; representing regions associated to three or more clusters over this five census period. Using the 'Add' mode for the trajectory selection, we select their trajectories, and a subset of the details is illustrated in Figure 7 . These regions account for about $5 \%$ of Toronto's population. The whole region was classified into the orange cluster in 1971 (low level of university degrees). By 1991, most of the region was classified into the green cluster, representing anglophone population, mostly Canadian born, with a higher level of education. As the corresponding plot indicates, this trend in increasing education is city-wide, but this region has people with better education than most.

In 2001, the purple cluster of high income annexes neighboring parts of the volatile region, and the Asian born population increases sharply, as illustrated by the appearance of the yellow cluster. This cluster indicates well educated, higher income, and about 30\%-50\% Asian born population. By 2011, the yellow cluster increased considerably, annexing parts of the high income purple cluster, including the neighboring Bridle Path area.

\subsection{Los Angeles}

We selected a region around the metropolitan area of Los Angeles (LA), following urban density. The summary of the results using all aspects with equal weights and eight 


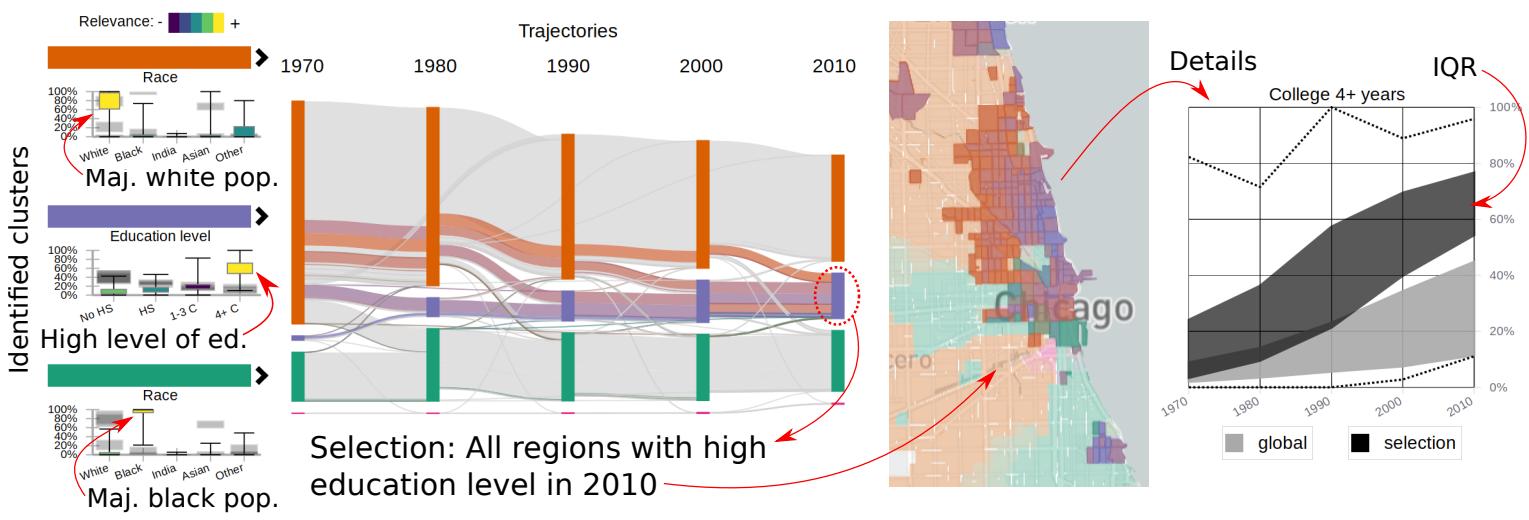

Fig. 5. Workflow to discover gentrification in Chicago: the purple cluster corresponds to high education / income. Its population is increasing over time, absorbing from the majority White cluster (orange). By selecting the purple cluster in 2010 , the region is highlighted in the maps. The proportion of people with $4+$ years of college is increasing in the whole city (grey IQRs), but significantly more in this region (black).

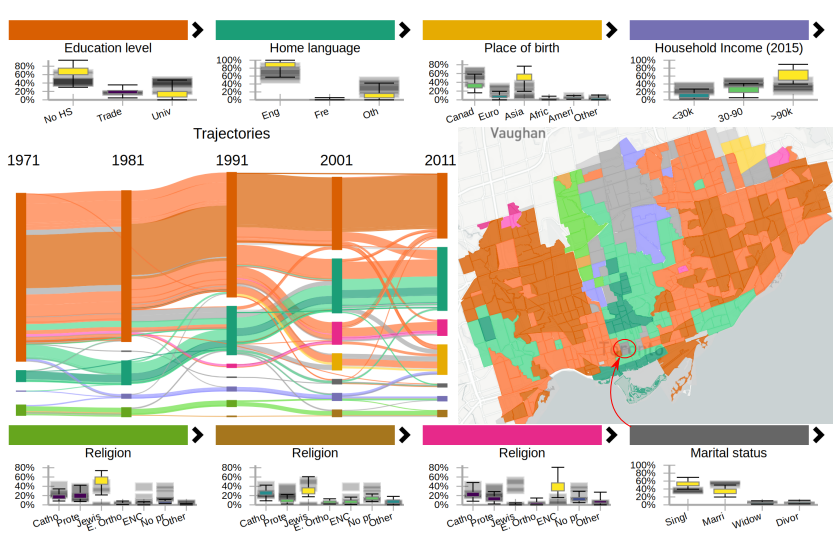

Fig. 6. Clustering results for Toronto, with eight clusters, including clusters representing Jewish population, high and low income, low education, and Asian immigration.

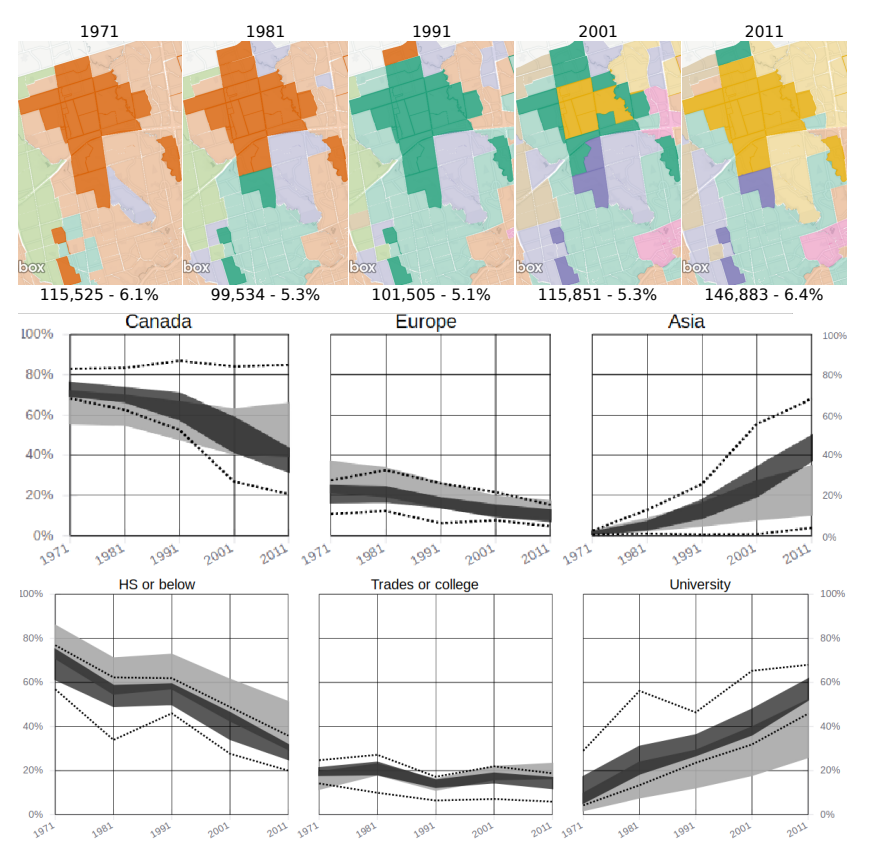

Fig. 7. Details for some regions of Toronto that were classified into 3 or more clusters over time.

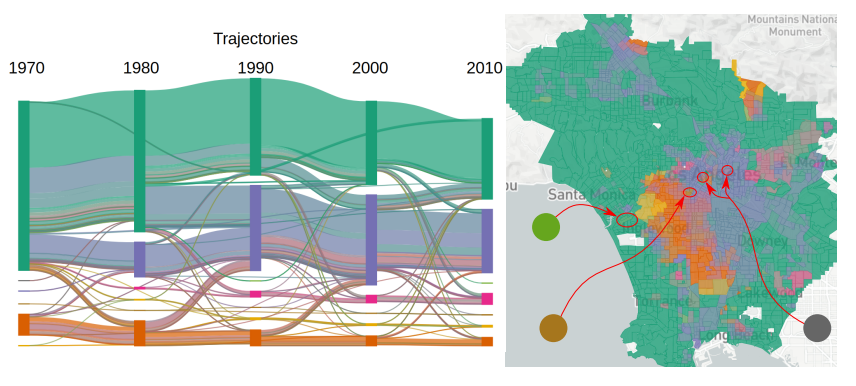

Fig. 8. Result for Los Angeles with 8 clusters, including three small and ephemeral clusters. Cluster characterization is displayed in Figure 9

clusters is illustrated in Figure 8 . The full statistical description of the clusters is illustrated in Figure 9, where the most relevant aspect of each cluster is highlighted. From the trajectories plot, we can see that there is a large but shrinking cluster, depicted in green, one increasing cluster in purple, an almost constant orange cluster, a smaller but increasing pink cluster, and three other small clusters. The corresponding map illustrates where these clusters are located, and that they are somewhat geographically stable, with some movement between the green, orange, and purple clusters.

From Figure 9, we can see that the green cluster is characterized by a high percentage of White population, low percentage of population in the lowest income bracket, mostly administrative occupations, and about $30 \%$ of the population with four or more years of college. The orange cluster is characterized by a high percentage of Black population, with few people in the highest range of income and education. The purple cluster corresponds to a high concentration of "Other" in race, which includes Hispanic for this dataset, high concentration of Laborers, and low education and income. The pink cluster contains a high percentage of Asian population and about $30 \%$ of the population with four or more years of college. The light green cluster contains very few people in the lower income bracket, mostly White population, with the highest percentage of population with four or more years of college, working administrative jobs, and a high concentration of singles. The yellow cluster represents Black population, with higher level of education and income, mostly working administrative jobs. The brown 
cluster represent a majority of single population, working administrative jobs with mostly low income. The dark gray cluster is characterized by all its population in the lowest income bracket, low education level, with a majority of White population. Since the extremes in the boxplots of the grey cluster are not significantly different, we can also surmise that this cluster is either small or homogeneous.

The green, orange, and purple clusters present a significant intra-cluster variance in most variables, as indicated by extreme whiskers of the boxplots. While fifty percent of the CTs in the green cluster have between $20 \%$ and $40 \%$ of people in the lowest income bracket, that cluster also includes CTs where none and all the population belongs to that bracket. This might indicate that this cluster represents different groups of people that are not different enough to be separated at this level of the hierarchy. Conversely, the light green, brown, and dark gray clusters are different enough to be separated into their own clusters at this level, despite being small and ephemeral, including only a few CTs.

The orange area in the map in Figure 8 presents movement, indicated by the presence of green and purple tones mixed with the orange, which may warrant further exploration. By clicking on the orange bar above the boxplot, we select all trajectories that contain the orange cluster. The corresponding details are illustrated in Figure 10. This shows a location change, where the orange cluster is progressively replaced by the purple cluster on its east side, and in turn expanding to the west. Interestingly, the population increased, the racial profile changed, but the distribution of income was reasonably stable, with a higher amount of the population in the lowest income range and very few people in the highest income range. Indeed, the income difference is significant when compared to the city-wide distribution.

A portion of this region is classified into the green cluster in 2010, indicating a majority white population. To further understand that change, we clear the current selection, and select all regions that changed from orange in 1970 to green in 2010, using the transition matrix. A portion of the resulting region, near the Florence-Graham region, is depicted in Figure 11. along with the temporal evolution of Race. Despite this difference, the other aspects are similar to the ones from the region in Figure 10, with slightly lower income and education profiles. While the racial aspect changed considerably, the economic and educational aspects stayed the same.

While Toronto is more dynamic than Los Angeles, possibly due to size differences, the volatile regions shown in Figure 7 did not change as quickly or dramatically as the ones shown in Figure 11, which involved twice as many people. We found this trend to be related to the countries themselves, Canadian cities have larger areas undergoing slow, gradual changes, whereas American cities have more general stability, but quicker changes in smaller scales. The supplementary material contains brief summaries of all the regions accessible in this prototype.

\section{EXPERT FEEDBACK}

We contacted academic and industry experts in sociology and urban sciences for their appreciation of our methodology. They had access to the prototype tool, a descriptive documentation of the features (included in the supplementary material), and a sequence of documentation videos illustrating how to perform specific tasks. The documentation explains which datasets are used, how the data is represented and processed, including that there is no geographic harmonization. We focused our inquires on the results obtained, asking if they found anything interesting on the data. The message sent and their full response is included in the supplementary material. Each of the five experts is identified by a letter, from A to E.

Their overall response was positive, mentioning that the prototype allows them to analyze census data without the additional work of obtaining and cleaning the data (A, B, E), and it allows the inclusion of geographic visual analysis tools in their research process (D). It enables the users to tell different stories about neighborhoods/cities and their changes (A), visualize the relationship between key urban variables over time (D), offering a quick way to identify particular neighborhoods that one may be interested in studying more in depth around a particular issue or efficiently understanding the context of an area (E). Indeed, the experts identified gentrification processes in Manhattan (B) and Dallas (E), reinforced a hypothesis for occupational clustering (D), and highlighted how the method can be used to compare neighborhoods and cities (A). In summary, the proposed methodology can be a viable alternative for the visual analytics of evolving demographic data.

The interface was "easy to navigate" (B), but it was also considered "overwhelming" (A), "intimidating" (E), and "tricky to interpret" (C), possible side-effects of our effort to increase representational accuracy, where we avoided using simplified representation or labels. Identifying clusters by their most relevant variables was welcome, but the overlap of information from different clusters in the boxplot was "a bit confusing" (C) when color was not present. Further, most clusters can be sufficiently characterized using only the most relevant aspect, but this is not generally true.

While the map of trajectories was mentioned as a "good summary map", how it related to the clustering method was unclear (C). The methods includes different options on how the colors are used, but both are sub optimal since reliably representing several distinct entities using colors is humanly unfeasible. Indeed, the number of distinguishable colors was a significant constraint, we found indications that more clusters should be used in some cases, even if eight clusters is more than what is traditionally considered in these analyses. Conversely, increasing the number of clusters would also complicate the interpretation of the results.

The experts also mentioned the poor responsiveness of the method when changes in the clustering parameters required server-side processing $(\mathrm{B}, \mathrm{D})$. Indeed, the current implementation can take a few minutes to cluster regions with high number of CTs, like Los Angeles or Brooklyn. Server-processing reduced the amount of data transferred to client, but it might increase the response time under load. We implemented a cache policy that greatly improved the performance, but fully pre-processing the results is not practical due to size of the parameter space.

Most of the experts demonstrated interest in using our method in their research (A, B, D, E), aiming to use the census data as a backdrop for other datasets, providing 


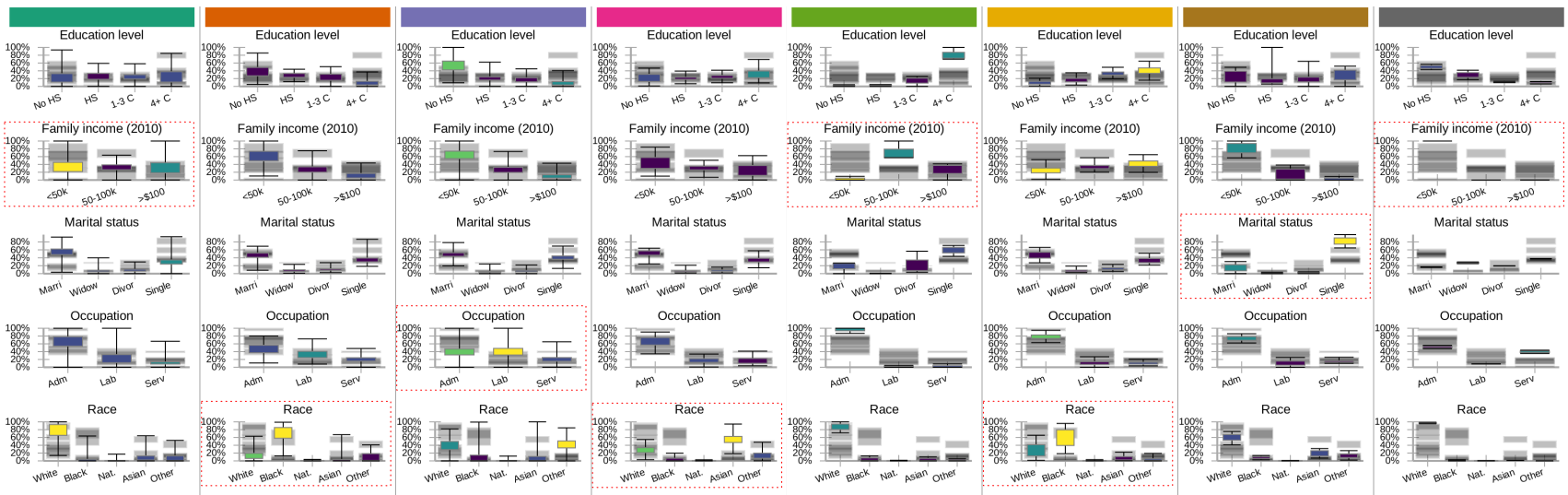

Fig. 9. Full characterization of the eight clusters found for LA. The red rectangles indicate the most relevant aspects for each cluster.

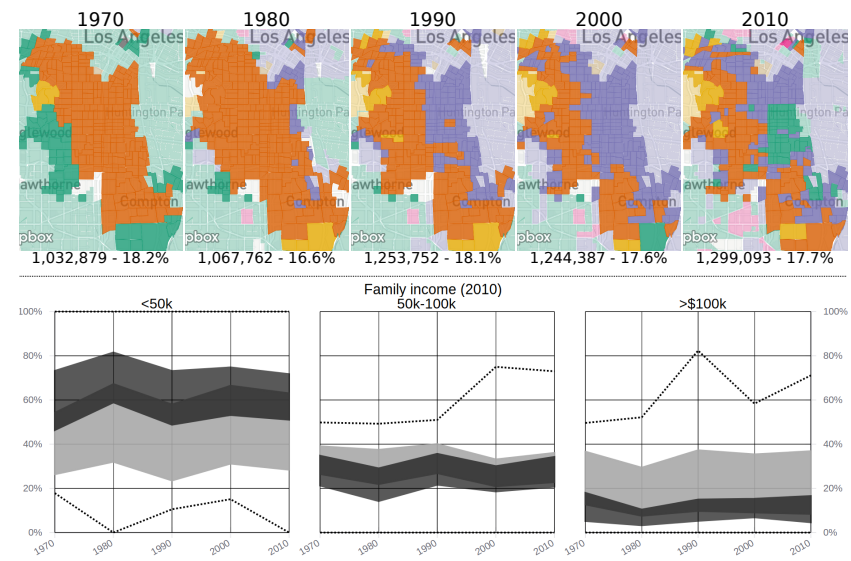

Fig. 10. Top: Geographic changes in the majority Black population cluster (orange) and Laborers cluster (green). Bottom: Income evolution for this region (black) and the whole city (gray).

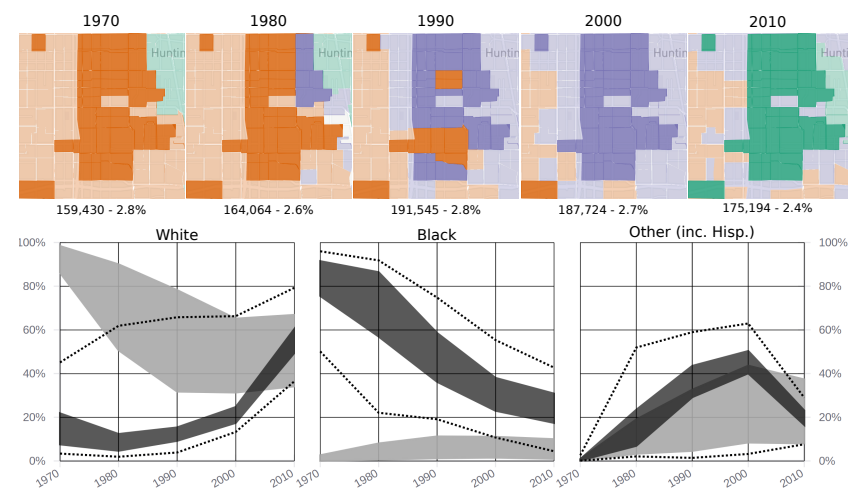

Fig. 11. Details for a volatile region contained in the area of Figure 10 This region went from Black to Hispanic to White.

demographic context. They also mentioned the need to export subsets of data, plots, and maps to be used in reports and publications (C, D, E). More importantly, while these experts were aware that our method does not perform geographic harmonization, none of them mention it. We did not specifically ask if this difference led to unexpected results, but rather if they found interesting insights. Most experts found phenomena corroborated by the specialized literature, indicating that our methodology produces equiv- alent results, with a fraction of the effort. We interpret the fact that most of them were interested in the next steps as confirmation of the accuracy of the method.

\section{Discussion AND LIMITATIONS}

Our objective was to leverage a graph based data representation and visualization methods for the exploration of geographically inconsistent region-based data. While we successfully replicated and corroborated results from the literature, this method still has significant limitations.

Removing the need for geographical harmonization greatly reduces the amount of work necessary to explore demographic data, but the method still requires consistent variables across the years. Matching the variables can be trivial for some aspects (Age), but challenging for others (Income). The divulged income ranges vary over time and the actual values change due to inflation. Some variables were not considered in earlier censuses, such as Race in Canada, or Hispanic population in the USA, hampering its use when they are available. Since this is only a prototype, we matched few aspects, but a proper demographic analysis would benefit from all available information.

While using one small map for each year leads to an easier visualization that does not require interaction, it does not scale if more than five or six years are considered. In this case, it might be interesting to replace the larger map considering each year individually, along with a temporal control for navigation. Indeed, including more years would likely lead to a stronger mixture of colors in the trajectory map, leading to a predominantly grey hue.

The limitation on the number of displayed clusters because of the limited number of distinguishable colors was significant. While increasing the number of clusters would further complicate an already complex analysis, it might be warranted for some regions. Color is a fundamental and intuitive tool for information representation that can be coherently used across different plots, so we opted to use it, even if in a limited way. With eight colors, there was overlap between some clusters, the relevance gradient, and the color combination.

Another limitation is the lack of control on how much the geographical information will impact the clustering result. While the adopted method met our needs for this work, 
a configurable control would add another dimension to the exploration, allowing for more intra-cluster variance to obtain more 'compact' clusters. We explored changing the number of content based augmented edges, but this proved to be unreliable and hard to interpret. The ClustGeo method [33] can be a viable option for this, allowing a graph based input and a hierarchical output, combined using a single mixing parameter. Alternatively, one could cluster the changes [56] instead of the stable states.

There are also technological limitations, such as memory use on the visualization client. To allow for changes on the CTs over the years, we use a geographic file that contains all possible intersections, which can grow rather large if the original city was expansive and contained several CTs, like NYC or LA. However, the most significant technological limitation relates to parameters that are not immediately interactive, such as the clustering configuration. Since the clustering is computationally expensive and performed on the server, which allows for cached results, some changes can take a few minutes to be considered, removing any possibility of a continuous exploration.

Indeed, the cognitive load on the user is already significant, as we compromised simplicity for accuracy. While other works labelled the clusters, as 'young urban', 'struggling', and so on [29], [30], we show the statistical characteristics of the clusters, which are harder to interpret, as the data may have subtle nuances that labels would otherwise hide. This also led to a crowded interface, mitigated somewhat the use of pop-up panels and collapsible sections. For some cities, especially if they are small and stable, the panels can appear redundant, but each provide a different way to interact with the information that can ease the exploration process for larger and dynamic cities.

\section{Conclusion}

Our objective was to allow for the exploration of census data without geographical harmonization, an original alternative to a challenging and error-prone process. Our method was able to corroborate previous findings from the specialized literature, with an increased level of detail due to our data representation and visualization choices. The feedback from experts was positive and most of them were able to extract insight from the prototype and demonstrated interest in using it on their research efforts. Indeed, the experts also demonstrated further interest in similar tools, indicating that visual analytics methods can be valuable in this field.

\section{ACKNOWLEDGEMENTS}

This research was supported by a University of Toronto Connaught Global Challenge grant and is part of the Urban Genome Project. The authors thank Cary Wu, Ethan Fosse, Fernando Caldern Figueroa, Patrick Adler, and James Murdoch for their expert opinions; Mark S. Fox, Robert M. Wright, Ultan Byrne, Matti Siemiatycki, Shauna Brail, and Richard Florida for general guidance and support; and the anonymous reviewers for their constructive comments.

\section{References}

[1] W. Chen, Z. Huang, F. Wu, M. Zhu, H. Guan, and R. Maciejewski, "Vaud: A visual analysis approach for exploring spatio-temporal urban data," IEEE Transactions on Visualization \& Computer Graphics, no. 1, pp. 1-1, 2017.

[2] T. Von Landesberger, F. Brodkorb, P. Roskosch, N. Andrienko, G. Andrienko, and A. Kerren, "Mobilitygraphs: Visual analysis of mass mobility dynamics via spatio-temporal graphs and clustering," IEEE transactions on visualization and computer graphics, vol. 22, no. 1, pp. 11-20, 2016.

[3] W. Chen, F. Guo, and F.-Y. Wang, "A survey of traffic data visualization," IEEE Transactions on Intelligent Transportation Systems, vol. 16, no. 6, pp. 2970-2984, 2015.

[4] S. Manson, J. Schroeder, D. V. Riper, and S. Ruggles. (2017) Ipums national historical geographic information system: Version 12.0 [database]. Minneapolis: University of Minnesota. [Online]. Available: http://doi.org/10.18128/D050.V12.0

[5] J. R. Logan, Z. Xu, and B. J. Stults, "Interpolating us decennial census tract data from as early as 1970 to 2010: A longitudinal tract database," The Professional Geographer, vol. 66, no. 3, pp. 412420, 2014.

[6] E. Hallisey, E. Tai, A. Berens, G. Wilt, L. Peipins, B. Lewis, S. Graham, B. Flanagan, and N. B. Lunsford, "Transforming geographic scale: a comparison of combined population and areal weighting to other interpolation methods," International Journal of Health Geographics, vol. 16, no. 1, p. 29, Aug 2017.

[7] J. Allen and Z. Taylor, "A new tool for neighbourhood change research: The canadian longitudinal census tract database, 19712016," The Canadian Geographer / Le Gographe canadien, vol. 0, no. 0, 2018. [Online]. Available: https: //onlinelibrary.wiley.com/doi/abs/10.1111/cag.12467

[8] J. R. Logan, B. J. Stults, and Z. Xu, "Validating population estimates for harmonized census tract data, 2000-2010," Annals of the American Association of Geographers, vol. 106, no. 5, pp. 1013-1029, 2016.

[9] C. L. Eicher and C. A. Brewer, "Dasymetric mapping and areal interpolation: Implementation and evaluation," Cartography and Geographic Information Science, vol. 28, no. 2, pp. 125-138, 2001.

[10] A. Dmowska, T. F. Stepinski, and P. Netzel, "Comprehensive framework for visualizing and analyzing spatio-temporal dynamics of racial diversity in the entire united states," PLOS ONE, vol. 12, no. 3, pp. 1-20, 032017.

[11] G. Firebaugh and C. R. Farrell, "Still large, but narrowing: The sizable decline in racial neighborhood inequality in metropolitan america, 1980-2010," Demography, vol. 53, no. 1, pp. 139-164, Feb 2016. [Online]. Available: https://doi.org/10.1007/ s13524-015-0447-5

[12] A. V. Diez-Roux, F. J. Nieto, C. Muntaner, H. A. Tyroler, G. W. Comstock, E. Shahar, L. S. Cooper, R. L. Watson, and M. Szklo, "Neighborhood environments and coronary heart disease: a multilevel analysis," American journal of epidemiology, vol. 146, no. 1, pp. 48-63, 1997.

[13] C. A. Gotway and L. J. Young, "Combining incompatible spatial data," Journal of the American Statistical Association, vol. 97, no. 458, pp. 632-648, 2002.

[14] X. Liu, Y. Song, K. Wu, J. Wang, D. Li, and Y. Long, "Understanding urban china with open data," Cities, vol. 47, pp. 53 - 61, 2015, current Research on Cities (CRoC).

[15] A. C.-D. Lee and C. Rinner, "Visualizing urban social change with self-organizing maps: Toronto neighbourhoods, 1996-2006," Habitat International, vol. 45, pp. 92-98, 2015.

[16] A. Dmowska and T. F. Stepinski, "Spatial approach to analyzing dynamics of racial diversity in large u.s. cities: 199020002010," Computers, Environment and Urban Systems, vol. 68, pp. 89 - 96, 2018. [Online]. Available: http://www.sciencedirect.com/ science/article/pii/S019897151730371X

[17] H. Doraiswamy, N. Ferreira, T. Damoulas, J. Freire, and C. T. Silva, "Using topological analysis to support event-guided exploration in urban data," IEEE Transactions on Visualization and Computer Graphics, vol. 20, no. 12, pp. 2634-2643, Dec 2014.

[18] D. I. Shuman, S. K. Narang, P. Frossard, A. Ortega, and P. Vandergheynst, "The emerging field of signal processing on graphs: Extending high-dimensional data analysis to networks and other irregular domains," IEEE Signal Processing Magazine, vol. 30, no. 3, pp. 83-98, 2013. 
[19] A. Sandryhaila and J. M. Moura, "Discrete signal processing on graphs," IEEE transactions on signal processing, vol. 61, no. 7, pp. 1644-1656, 2013.

[20] P. Valdivia, F. Dias, F. Petronetto, C. T. Silva, and L. G. Nonato, "Wavelet-based visualization of time-varying data on graphs," in 2015 IEEE Conference on Visual Analytics Science and Technology (VAST), Oct 2015, pp. 1-8.

[21] F. Dias and L. G. Nonato, "Some operators from mathematical morphology for the visual analysis of georeferenced data," in Workshop on Visual Analytics, Information Visualization and Scientific Visualization - SIBGRAPI, 2015.

[22] A. Dal Col, P. Valdivia, F. Petronetto, F. Dias, C. T. Silva, and L. G. Nonato, "Wavelet-based visual analysis of dynamic networks," IEEE Transactions on Visualization and Computer Graphics, vol. PP, no. 99, pp. 1-1, 2018.

[23] X. Huang, Y. Zhao, C. Ma, J. Yang, X. Ye, and C. Zhang, “Trajgraph: A graph-based visual analytics approach to studying urban network centralities using taxi trajectory data," IEEE Transactions on Visualization and Computer Graphics, vol. 22, no. 1, pp. 160-169, Jan 2016.

[24] C. Vehlow, F. Beck, and D. Weiskopf, "The State of the Art in Visualizing Group Structures in Graphs," in Eurographics Conference on Visualization (EuroVis) - STARs, R. Borgo, F. Ganovelli, and I. Viola, Eds. The Eurographics Association, 2015.

[25] F. Beck, M. Burch, S. Diehl, and D. Weiskopf, "The State of the Art in Visualizing Dynamic Graphs," in EuroVis - STARs, R. Borgo, R. Maciejewski, and I. Viola, Eds. The Eurographics Association, 2014.

[26] T. Setiadi, A. Pranolo, M. Aziz, S. Mardiyanto, B. Hendrajaya, and Munir, "A model of geographic information system using graph clustering methods," in 2017 3rd International Conference on Science in Information Technology (ICSITech), Oct 2017, pp. 727-731.

[27] A. Fahad, N. Alshatri, Z. Tari, A. Alamri, I. Khalil, A. Y. Zomaya, S. Foufou, and A. Bouras, "A survey of clustering algorithms for big data: Taxonomy and empirical analysis," IEEE Transactions on Emerging Topics in Computing, vol. 2, no. 3, pp. 267-279, sep 2014. [Online]. Available: https://doi.org/10.1109/tetc.2014.2330519

[28] A. K. Jain, "Data clustering: 50 years beyond k-means," Pattern recognition letters, vol. 31, no. 8, pp. 651-666, 2010.

[29] E. C. Delmelle, "Mapping the dna of urban neighborhoods: Clustering longitudinal sequences of neighborhood socioeconomic change," Annals of the American Association of Geographers, vol. 106, no. 1, pp. 36-56, 2016.

[30] — "Differentiating pathways of neighborhood change in 50 u.s. metropolitan areas," Environment and Planning A: Economy and Space, vol. 49, no. 10, pp. 2402-2424, 2017.

[31] C. Ling and E. C. Delmelle, "Classifying multidimensional trajectories of neighbourhood change: a self-organizing map and kmeans approach," Annals of GIS, vol. 22, no. 3, pp. 173-186, 2016.

[32] J. Han, M. Kamber, and A. K. Tung, "Spatial clustering methods in data mining," Geographic data mining and knowledge discovery, pp. 188-217, 2001.

[33] M. Chavent, V. Kuentz-Simonet, A. Labenne, and J. Saracco, "Clustgeo: an r package for hierarchical clustering with spatial constraints," Computational Statistics, pp. 1-24, 2017.

[34] S. Soor, A. Challa, S. Danda, B. D. Sagar, and L. Najman, "Extending k-means to preserve spatial connectivity," 2018.

[35] P. Soille and L. Najman, "On morphological hierarchical representations for image processing and spatial data clustering," in Applications of Discrete Geometry and Mathematical Morphology. Springer, 2012, pp. 43-67.

[36] J. Cousty, G. Bertrand, L. Najman, and M. Couprie, “Watershed cuts: Minimum spanning forests and the drop of water principle," IEEE Transactions on Pattern Analysis and Machine Intelligence, vol. 31, no. 8, pp. 1362-1374, Aug 2009.

[37] M. D. Dias, M. R. Mansour, F. Dias, F. Petronetto, C. T. Silva, and L. G. Nonato, "A hierarchical network simplification via nonnegative matrix factorization," in 2017 30th SIBGRAPI Conference on Graphics, Patterns and Images (SIBGRAPI), Oct 2017, pp. 119-126.

[38] M. Monmonier, "Strategies for the visualization of geographic time-series data," Cartographica: The International Journal for Geographic Information and Geovisualization, vol. 27, no. 1, pp. 30-45, 1990.

[39] N. Andrienko, G. Andrienko, and P. Gatalsky, "Exploratory spatiotemporal visualization: an analytical review," Journal of Visual Languages \& Computing, vol. 14, no. 6, pp. 503-541, 2003.
[40] N. Ferreira, "Visual analytics techniques for exploration of spatiotemporal data," Ph.D. dissertation, Polytechnic Institute of New York University, 2015.

[41] Y. Zheng, W. Wu, Y. Chen, H. Qu, and L. M. Ni, "Visual analytics in urban computing: An overview," IEEE Transactions on Big Data, vol. 2, no. 3, pp. 276-296, Sept 2016.

[42] M. O. Ward, G. Grinstein, and D. Keim, Interactive data visualization: foundations, techniques, and applications. AK Peters/CRC Press, 2015

[43] G. Andrienko, N. Andrienko, H. Schumann, and C. Tominski, "Visualization of trajectory attributes in space-time cube and trajectory wall," in Cartography from Pole to Pole. Springer, 2014, pp. 157-163.

[44] C. Tominski and H.-J. Schulz, "The Great Wall of Space-Time," in Vision, Modeling and Visualization, M. Goesele, T. Grosch, H. Theisel, K. Toennies, and B. Preim, Eds. The Eurographics Association, 2012.

[45] S. Buschmann, M. Trapp, and J. Döllner, "Real-time animated visualization of massive air-traffic trajectories," in Cyberworlds (CW), 2014 International Conference on. IEEE, 2014, pp. 174-181.

[46] D. Seebacher, J. Häußler, M. Hundt, M. Stein, H. Müller, U. Engelke, and D. Keim, "Visual analysis of spatio-temporal event predictions: Investigating the spread dynamics of invasive species," in 2017 IEEE Visualization Conference (VIS), 2017.

[47] G. Andrienko, N. Andrienko, G. Fuchs, and J. Wood, "Revealing patterns and trends of mass mobility through spatial and temporal abstraction of origin-destination movement data," IEEE Transactions on Visualization and Computer Graphics, vol. 23, no. 9, pp. 2120 2136, Sept 2017.

[48] N. Ferreira, M. Lage, H. Doraiswamy, H. Vo, L. Wilson, H. Werner, M. Park, and C. Silva, "Urbane: A 3d framework to support data driven decision making in urban development," in Visual Analytics Science and Technology (VAST), 2015 IEEE Conference on. IEEE, 2015, pp. 97-104.

[49] M. Li, Z. Bao, T. Sellis, S. Yan, and R. Zhang, "Homeseeker: A visual analytics system of real estate data," Journal of Visual Languages \& Computing, vol. 45, pp. 1 - 16, 2018.

[50] J. Johansson, P. Ljung, M. Jern, and M. Cooper, "Revealing structure within clustered parallel coordinates displays," in Information Visualization, 2005. INFOVIS 2005. IEEE Symposium on. IEEE, 2005, pp. $125-132$.

[51] D. Guo, J. Chen, A. M. MacEachren, and K. Liao, "A visualization system for space-time and multivariate patterns (vis-stamp)," IEEE transactions on visualization and computer graphics, vol. 12, no. 6, pp. 1461-1474, 2006.

[52] F. Pedregosa, G. Varoquaux, A. Gramfort, V. Michel, B. Thirion, O. Grisel, M. Blondel, P. Prettenhofer, R. Weiss, V. Dubourg, J. Vanderplas, A. Passos, D. Cournapeau, M. Brucher, M. Perrot, and E. Duchesnay, "Scikit-learn: Machine learning in Python," Journal of Machine Learning Research, vol. 12, pp. 2825-2830, 2011.

[53] M. Harrower and C. A. Brewer, "Colorbrewer.org: An online tool for selecting colour schemes for maps," The Cartographic Journal, vol. 40, no. 1, pp. 27-37, 2003.

[54] J. L. Hintze and R. D. Nelson, "Violin plots: a box plot-density trace synergism," The American Statistician, vol. 52, no. 2, pp. 181$184,1998$.

[55] J. Hwang and R. J. Sampson, "Divergent pathways of gentrification: Racial inequality and the social order of renewal in chicago neighborhoods," American Sociological Review, vol. 79, no. 4, pp. 726-751, 2014

[56] J. Bian, D. Tian, Y. Tang, and D. Tao, "A survey on trajectory clustering analysis," arXiv preprint arXiv:1802.06971, 2018. 